\title{
The spectrum of the recurrent nova U Scorpii during the 1999 outburst
}

\begin{abstract}
T. Iijima*
Astronomical Observatory of Padova, Asiago Section, Osservatorio Astrofisico, 36012 Asiago (Vi), Italy

Received 10 May 2001 / Accepted 25 February 2002

Abstract. An optical spectrum covering the range $\lambda \lambda 4300-6900 \AA$ of the recurrent nova U Sco in outburst was taken on February 26th 1999, only 16 hours after maximum. Broad emission lines of H I, He I, N II, N III were seen on the spectrum, while the emission line of He II $\lambda 4686$ was not yet seen. The $F W H M$ of the emission component of $\mathrm{H} \alpha$ was $8000 \mathrm{~km} \mathrm{~s}^{-1}$ and the $F W Z I$ was roughly $12000 \mathrm{~km} \mathrm{~s}^{-1}$. The emission line of $\mathrm{H} \alpha$ had blue $\left(-3250 \mathrm{~km} \mathrm{~s}^{-1}\right)$, central $\left(+30 \mathrm{~km} \mathrm{~s}^{-1}\right)$, and red $\left(+2770 \mathrm{~km} \mathrm{~s}^{-1}\right)$ peaks, among which the red one was the most prominent. A narrow absorption component of $\mathrm{H} \alpha$ blue-shifted by $-4850 \mathrm{~km} \mathrm{~s}^{-1}$ was seen. The other prominent lines had a main emission peak red-shifted by $2000-3000 \mathrm{~km} \mathrm{~s}^{-1}$ and a weak secondary peak blueshifted by $-2000--3000 \mathrm{~km} \mathrm{~s}^{-1}$. Most of He I and N II lines were flanked by a narrow absorption component blue-shifted by about $-4300 \mathrm{~km} \mathrm{~s}^{-1}$ which was a good clue for the detailed identification of these lines. These absorption components and some emission lines of N II faded very rapidly and nearly disappeared one day after our observation. The widths of the emission lines decreased with time. The radial velocities of three emission peaks of $\mathrm{H} \alpha$ were $-1560 \mathrm{~km} \mathrm{~s}^{-1},+60 \mathrm{~km} \mathrm{~s}^{-1}$ and $+1800 \mathrm{~km} \mathrm{~s}^{-1}$ on March 14th, 17 days after maximum, when the blue one became stronger than the others.

The helium abundance by the number of atom of the ejecta is estimated as $N(\mathrm{He}) / N(\mathrm{H})=0.16 \pm 0.02$ which is much lower than those found in previous works, and is not different from those of normal classical novae. Adopting the distance $6 \mathrm{kpc}$, the mass of the ejecta is estimated as $3-9 \times 10^{-7} M_{\odot}$.
\end{abstract}

Key words. stars: individual: U Sco - novae, cataclysmic variables

\section{Introduction}

The recurrent nova U Sco demonstrates extremely rapid photometric and spectroscopic developments in the outbursts (see, the review of Webbink et al. 1987). After 12 years of quiescence, this object underwent a new outburst in February 1999 (Schmeer 1999). The $V$ magnitude near maximum was 7.6 at February 25.562 UT (Shaw 1999 ) and 8.0 at 25.625 UT (Mattiazzo 1999). Preliminary reports of spectroscopic observations have been made by some astronomers (e.g. Bonifacio et al. 1999; Iijima 2000; Niedzielski et al. 1999; Zwitter \& Munari 1999). Lèpine et al. (1999) presented the results of optical spectroscopy made by HST, and Kahabka et al. (1999) detected soft X-ray emission during the outburst. Munari et al. (1999) reported large variations of the profile of the $\mathrm{H} \alpha$ emission line, in particular the decreasing of its width with time. Anupama \& Dewangan (2000) estimated the mass and helium abundance of the ejecta, but as discussed later, they analyzed spectra taken more then 10 days after light maximum. So, their results are somewhat different from those obtained in this work. Schaefer \& Ringwald (1995)

\footnotetext{
* e-mail: iijima@astras.pd.astro.it
}

showed that this object is an eclipsing binary system with an orbital period of 1.23 day, and Hachisu et al. (2000a) proposed a model of thermonuclear burning on a massive white dwarf for the outbursts.

We took a high dispersion spectrum of this object only 16 hours after maximum, showing spectral features very different from those observed in the previous outbursts (e.g. Barlow et al. 1981; Rosino \& Iijima 1988; Sekiguchi et al. 1988). The differences may be mainly due to the timing of the observations, because the first spectra in the previous outbursts were taken at least several days after maximum. Detailed descriptions of the spectra in the present outburst are given in this paper. The mass and helium abundance of the ejecta are estimated.

\section{Observation}

High dispersion spectra covering the range of $\lambda \lambda 4300$ $6900 \AA$ with a resolution $\lambda / \Delta \lambda=8000$ were taken with an Echelle spectrograph mounted on the $182 \mathrm{~cm}$ telescope at the Mount Ekar station of the Astronomical Observatory of Padova. A medium dispersion spectrum was taken with a Boller and Chivens grating spectrograph mounted on 
Table 1. Journal of observations.

\begin{tabular}{lrccrrrcc}
\hline \hline \multicolumn{2}{c}{\begin{tabular}{c} 
Date \\
\multicolumn{1}{c}{ 1999 }
\end{tabular}} & UT & JD & $\begin{array}{r}\Delta t \\
\text { day }\end{array}$ & $\begin{array}{r}\text { exp. } \\
\text { s }\end{array}$ & Inst. & $\begin{array}{c}\text { Range } \\
\AA\end{array}$ & $m_{V}$ \\
\hline Feb. & 26 & $5: 02$ & 1235.71 & 0.65 & 900 & Ech & $4300-6900$ & 8.8 \\
March & 8 & $4: 46$ & 1245.70 & 10.64 & 800 & B\&C & $4000-5100$ & 13 \\
March & 14 & $4: 04$ & 1251.67 & 16.61 & 3000 & Ech & $4300-6900$ & 14 \\
\hline
\end{tabular}

JD: Julian day -2450000 .

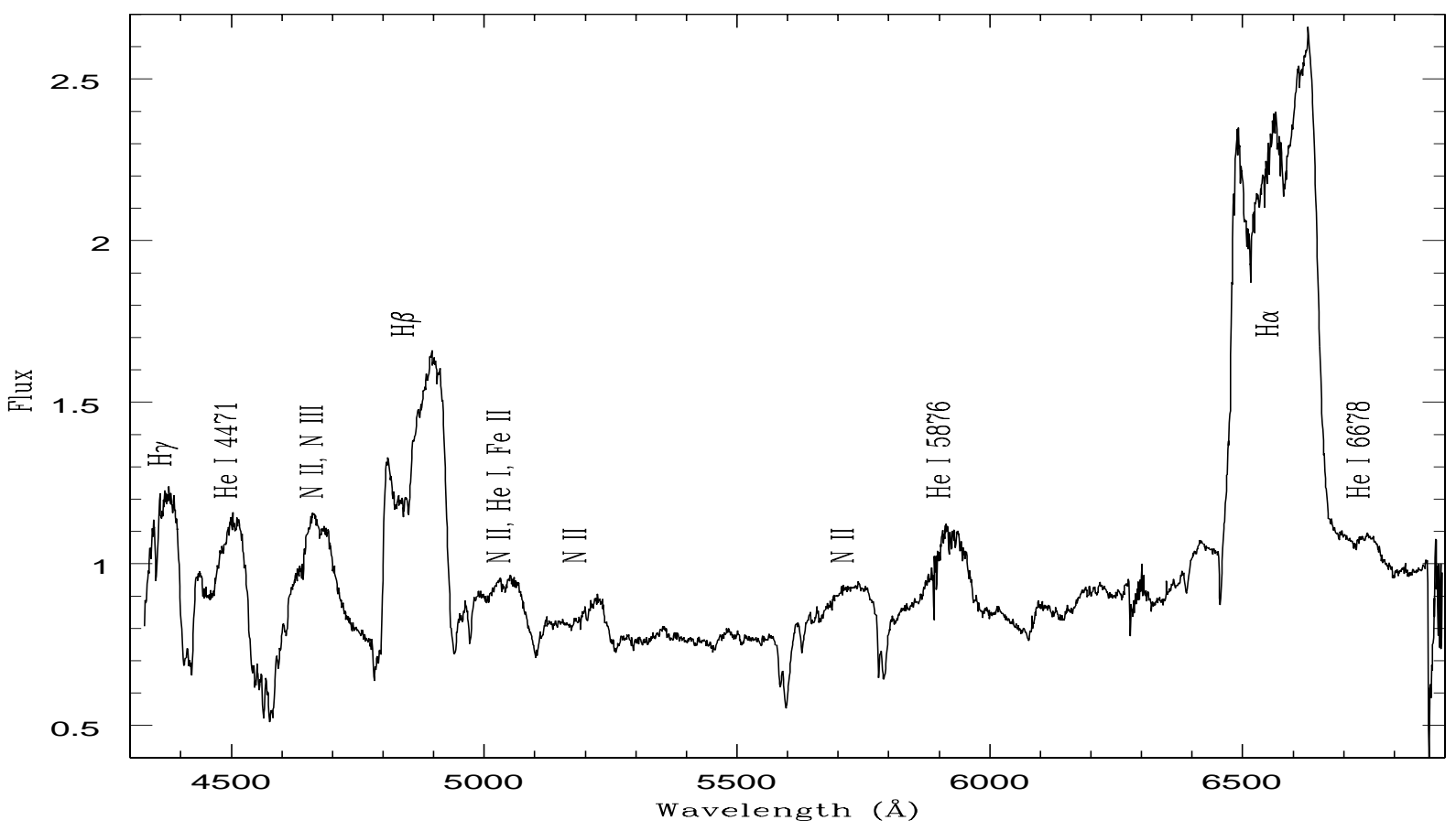

Fig. 1. A tracing of the spectrum of U Sco taken on 1999 February 26.21 UT, 16 hours after maximum. The unit of the ordinate is $10^{-12} \mathrm{erg} \mathrm{cm}^{-2} \mathrm{~s}^{-1} \AA^{-1}$. Detailed identification of the features is presented in Figs. 2-5.

the $122 \mathrm{~cm}$ telescope of the Astrophysical Observatory of Asiago of the University of Padova. The data reductions were carried out using the NOAO IRAF package at Asiago Observatory. The spectrophotometric calibrations were made using the spectra of standard stars taken in the same nights. $58 \mathrm{Aql}$ and HD 60778 were used for the high dispersion spectra and Kopff 27 for the medium dispersion spectrum. The absolute calibration of the spectrum taken on February 26.21 UT was made comparing the integrated flux in the range $\lambda \lambda 5000-6200 \AA$, according to the spectral response of the $V$ band, with the observed $V$ magnitude at that time $\left(m_{V}=8.8\right.$ in VS-Net). A journal of the observations is given in Table 1 , where UT is the universal time at mid-exposure.

\section{Spectrum near maximum luminosity}

Our first spectrum was taken on February 26.21 UT (JD: 2451235.71), when the $V$ magnitude was about 8.8. A trace of the full spectrum is shown in Fig. 1, where the unit of the ordinate is $10^{-12} \mathrm{erg} \mathrm{cm}^{-2} \mathrm{~s}^{-1} \AA^{-1}$.

It was difficult to make a detailed identification of the emission features in the spectra at the early stage of explosion of this object, because they were broad and blended with each other. In our spectrum, however, numerous narrow absorption components are detected. As seen in Sect. 5, the electron density of the ejecta was of the order of $10^{10} \mathrm{~cm}^{-3}$ when the spectrum was taken. Therefore, the contribution from forbidden lines is negligible. The line identifications for our spectrum are made assuming that a principal emission component has a corresponding absorption component.

The wavelengths of the absorption components and most emission components are obtained with simple gaussian fittings. On the other hand, some emission components have asymmetric profiles, e.g. the red peak of $\mathrm{H} \alpha$. In such a case, the wavelength at the middle of the emission body is measured. The heliocentric radial velocities of the prominent emission and absorption features are summarized in Table 2. The observational errors are about $\pm 10 \mathrm{~km} \mathrm{~s}^{-1}$, but those of the broad emission features should be larger. The results with larger observational errors, probably larger than $\pm 30 \mathrm{~km} \mathrm{~s}^{-1}$, are denoted by a colon. The observed intensities of the prominent emission features are presented in Table 3 , where $F(\lambda)$ is the observed flux and $I(\lambda)$ is the intensity after correction for 
Table 2. Heliocentric radial velocities of emission and absorption features.

\begin{tabular}{|c|c|c|c|c|c|c|}
\hline element & $\lambda(\AA)$ & em1 & em2 & em3 & abs & blended by \\
\hline Feb. 26 & \multicolumn{5}{|c|}{$\left(\mathrm{km} \mathrm{s}^{-1}\right)$} & \\
\hline He I 14 & 4471 & +2180 & -2340 & & -4320 & $\operatorname{Mg}$ II 4, 4481 \\
\hline $\mathrm{H} \beta$ & 4861 & +2320 & -3160 & & -4820 & \\
\hline He I 48 & 4922 & & & & -4310 & Fe II 42,4924 \\
\hline N II 4 & 5011 & & & & $-4130:$ & He I 4,5016, Fe II 42,5018 \\
\hline N II 4 & 5045 & & & & -4310 & He I 47,5048 \\
\hline N II 66,70 & 5180 & +2470 & -3070 & & -4420 & N II 66,5176 \\
\hline N II 69 & 5321 & +1830: & -2360 : & & & \\
\hline N II 63 & 5535 & & -2740 & & -4240 & \\
\hline$[\mathrm{N} \mathrm{II}]^{*} 3 \mathrm{~F}$ & 5755 & +2720 & & & & \\
\hline N II 3 & 5667 & & -2500 & & -4250 & \\
\hline N II 3 & 5679 & $+2700:$ & -1850 & & -4320 & N II $3,5676,5686$ \\
\hline N II 3 & 5711 & & -2730 & & -4280 & \\
\hline He I 11 & 5876 & +2410 & $-2480:$ & & -4310 & \\
\hline N II 27 & 6066 & +1910 & & & & \\
\hline N II 36,60 & 6168 & +1930 & & & -4430 & \\
\hline N II 8 & 6482 & & -2880 & & -4280 & $\mathrm{H} \alpha$ \\
\hline $\mathrm{H} \alpha$ & 6563 & +2770 & -3250 & +30 & -4850 & \\
\hline He I 46 & 6678 & +3040: & & & & $\mathrm{H} \alpha$ \\
\hline March 8 & \multicolumn{5}{|c|}{$\left(\mathrm{km} \mathrm{s}^{-1}\right)$} & \\
\hline $\mathrm{H} \beta$ & 4861 & +1980 & -1600 & +80 & & \\
\hline $\mathrm{H} \alpha$ & 6563 & +1800 & -1560 & +60 & & \\
\hline
\end{tabular}

*: [N II] $\lambda 5755$ line was very weak and it is not sure whether it was really detected.

the interstellar extinction $E(B-V)=0.2$ (Barlow et al. 1981). The errors in the intensities of the prominent emission features are about $\pm 10 \%$ and values of lower accuracy are denoted by a colon. The intensities of the emission features located in the range bluer than $\lambda 4700 \AA$ are rather uncertain, because it is difficult to determine the precise continuum level.

Detailed descriptions of the different parts of the spectrum are given below.

\section{1. $H \alpha$ region}

Figure 2 shows the profile of $\mathrm{H} \alpha$. The FWHM of the emission is about $8000 \mathrm{~km} \mathrm{~s}^{-1}$. There are three emission peaks, among which the red one the most intense. The radial velocities of the individual peaks are $+2770 \mathrm{~km} \mathrm{~s}^{-1}$, $+30 \mathrm{~km} \mathrm{~s}^{-1}$ and $-3250 \mathrm{~km} \mathrm{~s}^{-1}$, respectively. Two narrow absorptions are seen at $\lambda \lambda 6457$ and $6389 \AA$. The former could be due to $\mathrm{H} \alpha$ blue-shifted by $-4850 \mathrm{~km} \mathrm{~s}^{-1}$, while the latter may depend on N II multiplet number 8 at $\lambda 6482 \AA$ (Moore 1959) blue-shifted by $-4280 \mathrm{~km} \mathrm{~s}^{-1}$. The weak emission hump at $\lambda 6420 \AA$ could be the secondary emission component of N II 8, $\lambda 6482$ blue-shifted by $-2880 \mathrm{~km} \mathrm{~s}^{-1}$. As seen later, these radial velocities are consistent with those of the other N II lines. The main emission component of this transition, which should be red-shifted by $2000-3000 \mathrm{~km} \mathrm{~s}^{-1}$, may be embedded in the emission component of $\mathrm{H} \alpha$. It is difficult to estimate

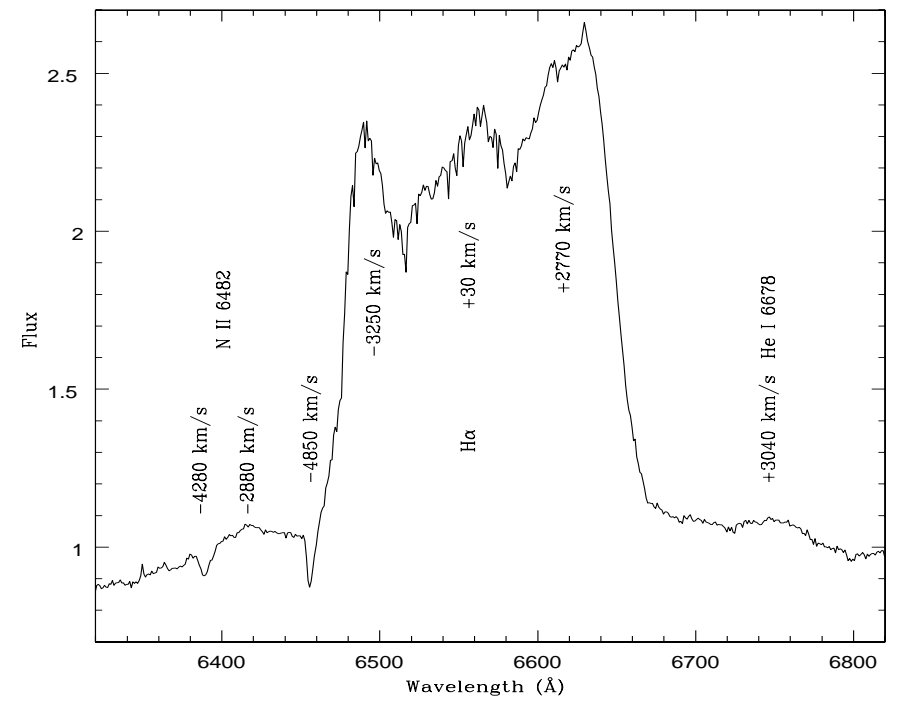

Fig. 2. $\mathrm{H} \alpha$ region.

the full width at zero intensity $(F W Z I)$ of $\mathrm{H} \alpha$, because its red-ward end is blended with the emission line of He I $\lambda 6678$, and the blue-ward tail is blended with N II $\lambda 6482$. A rough estimate of $F W Z I \sim 12000 \mathrm{~km} \mathrm{~s}^{-1}$ was made by eye. The radial velocity of the main emission component of He I $\lambda 6678$ is $+3040 \mathrm{~km} \mathrm{~s}^{-1}$. 


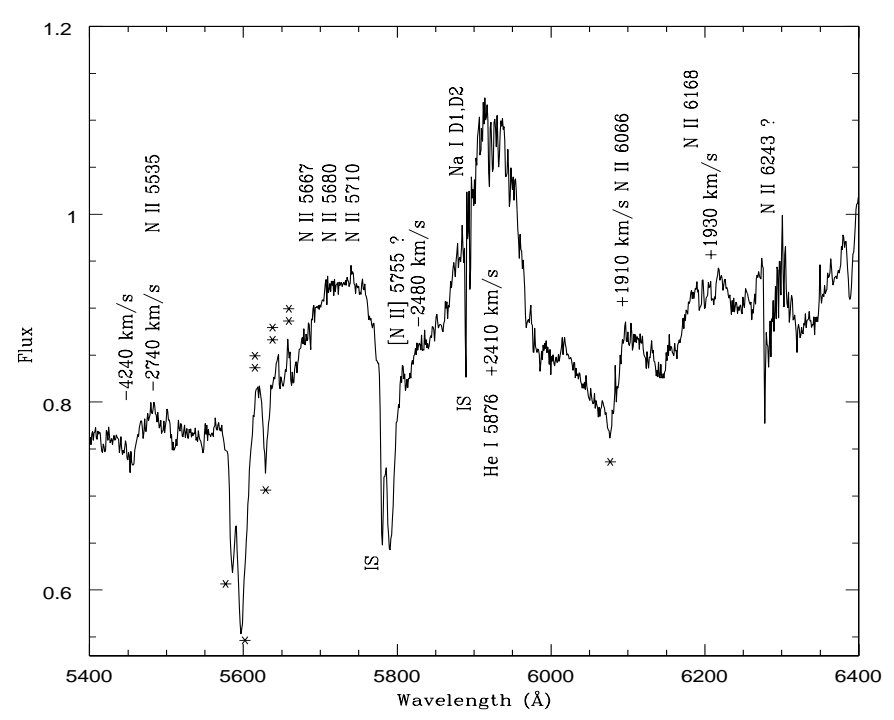

Fig. 3. The spectral region $5450-6400 \AA$.

\subsection{From $5450 \AA$ to $6400 \AA$}

Figure 3 shows the spectral region $\lambda \lambda 5450-6400 \AA$. It seems that there is an emission feature around $\lambda 6290 \AA$, which just coincides with the atmospheric $\mathrm{O}_{2}$ absorption band. Probably there is an emission component of N II 57, $\lambda 6242.5$ red-shifted by about $+2000 \mathrm{~km} \mathrm{~s}^{-1}$, but a detailed measurement is not possible.

The broad emission features at $\lambda \lambda 6100$ and $6200 \AA$ could be identified as N II 27, $\lambda 6065.5$ and N II 36,60, $\lambda 6167.8$ red-shifted by $1910 \mathrm{~km} \mathrm{~s}^{-1}$ and $1930 \mathrm{~km} \mathrm{~s}^{-1}$, respectively. The absorption feature at $\lambda 6076 \AA$, indicated by an asterisk, may be due to N II $36,60, \lambda 6167.8$ blueshifted by $-4430 \mathrm{~km} \mathrm{~s}^{-1}$. These emission and absorption features were seen also in a spectrum taken at 26.011 UT by Anupama \& Dewangan (2000), but faded very rapidly after our observation. Only their weak traces remained in a spectrum taken at 27.018 UT by Anupama \& Dewangan (2000), no trace was then seen at 27.188 UT (Munari et al. 1999).

The main emission component of He I $\lambda 5876$ is redshifted by $2410 \mathrm{~km} \mathrm{~s}^{-1}$. The secondary emission component of this element is very weak. The barely visible emission hump at $\lambda 5827 \AA$ could be identified as the secondary emission blue-shifted by $-2480 \mathrm{~km} \mathrm{~s}^{-1}$. The weak narrow emission line at $\lambda 5808 \AA$ might be identified as $[\mathrm{N} \mathrm{II}]$ $\lambda 5755$ red-shifted by $2720 \mathrm{~km} \mathrm{~s}^{-1}$. There are two absorption lines at $\lambda \lambda 5781$ and $5791 \AA$. The latter may be due to He I $\lambda 5876$ blue-shifted by $-4310 \mathrm{~km} \mathrm{~s}^{-1}$, while the former, indicated as IS, could be identified as the interstellar absorption band at $\lambda 5780.6 \AA$.

The equivalent widths of the interstellar absorption components of Na I D1 and D2, indicated as IS, are 0.26 and $0.33 \AA$, respectively.

There are three absorption features, indicated by an asterisk, at $\lambda \lambda 5586,5598$ and $5629 \AA$. The first one could be identified as N II $3, \lambda 5666.6$ blue-shifted by $-4250 \mathrm{~km} \mathrm{~s}^{-1}$, while the second may be a blend of
Table 3. Intensity of prominent emission features on 1999 Feb. $26.21 \mathrm{UT}$.

\begin{tabular}{cllrr}
\hline \hline $\begin{array}{c}\lambda_{\text {center }} \\
\AA\end{array}$ & $\begin{array}{l}f w h m \\
\mathrm{~km} \mathrm{~s}^{-1}\end{array}$ & elements & $F(\lambda)$ & $I(\lambda)$ \\
\hline 4488 & 3450 & He I 4471, Mg II & $2.3:$ & $4.6:$ \\
4670 & 4990 & N II, N III & $4.0:$ & $7.8:$ \\
4874 & 7630 & H $\beta$ & 9.0 & 17.0 \\
5026 & 7240 & N II, He I, Fe II & 2.5 & 5.0 \\
5192 & 6990 & N II, Fe II & 1.4 & 2.4 \\
5718 & 4650 & N II & 1.5 & 2.5 \\
5916 & 3410 & He I 5876 & 2.5 & 4.1 \\
6111 & $\ldots$ & N II & 0.3 & 0.4 \\
6207 & $\ldots$ & N II & 0.9 & 1.4 \\
6568 & 8000 & H $\alpha$ & 30.1 & 46.0 \\
6747 & $\cdots$ & He I 6678 & $0.9:$ & $1.3:$ \\
\hline
\end{tabular}

Unit of $F(\lambda)$ and $I(\lambda)$ is $10^{-11} \mathrm{erg} \mathrm{cm}^{-2} \mathrm{~s}^{-1}$.

N II 3, $\lambda \lambda 5676.0,5679.6$, and 5686.2. If the line of $\lambda 5679.6$ is the main contributor to the blend, its blue-shift is $-4320 \mathrm{~km} \mathrm{~s}^{-1}$. The third one may be due to $\mathrm{N}$ II 3 , $\lambda 5710.8$ blue-shifted by $-4280 \mathrm{~km} \mathrm{~s}^{-1}$. It is not possible to separate the red-shifted main emission components of these transitions. Since the center of the diffuse emission component is found at $\lambda 5730 \AA$, the red-shifts of the emission components may be roughly $+2700 \mathrm{~km} \mathrm{~s}^{-1}$. There are three weak emission features at $\lambda \lambda 5619,5644$, and $5658 \AA$, indicated by two asterisks. These features may be due to the secondary emission components of these transitions. Their radial velocities are $-2500 \mathrm{~km} \mathrm{~s}^{-1},-1850 \mathrm{~km} \mathrm{~s}^{-1}$, and $-2730 \mathrm{~km} \mathrm{~s}^{-1}$, respectively.

There are a weak emission feature at $\lambda 5484 \AA$ and an absorption at $\lambda 5457 \AA$. These features could be identified as the secondary emission component and the absorption component of N II $63, \lambda 5535.4$. If this is the case, their radial velocities are $-2740 \mathrm{~km} \mathrm{~s}^{-1}$ and $-4240 \mathrm{~km} \mathrm{~s}^{-1}$, respectively. In contrast to the other lines of $\mathrm{N}$ II, the primary emission component of this transition is not seen. If the primary emission component is red-shifted by about $3000 \mathrm{~km} \mathrm{~s}^{-1}$, it should just coincide with the deep absorption features at $\lambda \lambda 5586$ and $5598 \AA$. Probably this is the reason why the primary emission component is not seen.

The absorption components of the He I and N II lines also were clearly seen in a spectrum taken at 26.011 UT by Anupama \& Dewangan (2000), but disappeared at 27.018 UT (Anupama \& Dewangan 2000). On the other hand, the emission components lasted for several days after maximum (Sekiguchi et al. 1988; Munari et al. 1999).

\subsection{From $4950 \AA$ to $5500 \AA$}

Figure 4 shows the spectral region $\lambda \lambda 4950-5500 \AA$. The emission feature in the range $\lambda \lambda 5120-5230 \AA$ could be identified as $\mathrm{N}$ II $66, \lambda \lambda 5175.9$ and 5179.5 and $\mathrm{N}$ II $70, \lambda 5179.5$. If we use the wavelength $\lambda 5179.5$, the radial velocity of the main emission component is $+2470 \mathrm{~km} \mathrm{~s}^{-1}$, and that of the secondary component is 


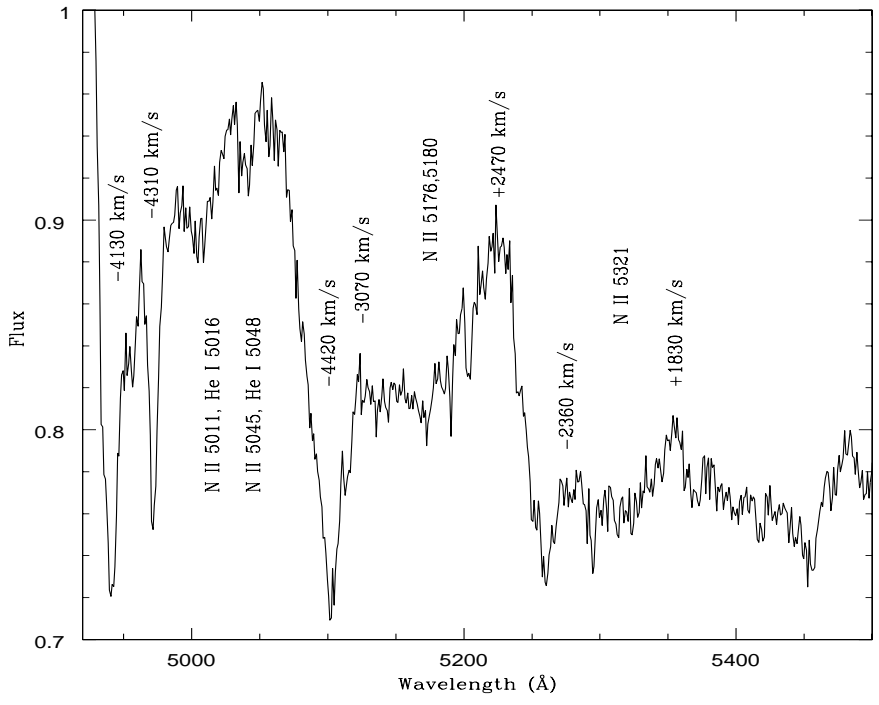

Fig. 4. The spectral region 4950-5500 ̊.

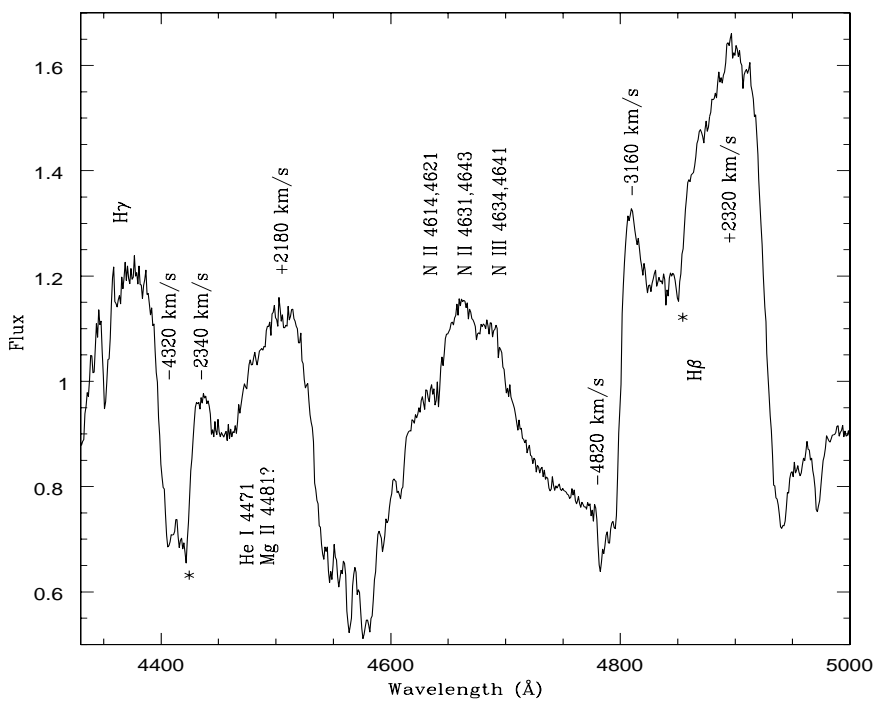

Fig. 5. The spectral region $4350-5000 \AA$.

$-3070 \mathrm{~km} \mathrm{~s}^{-1}$. The radial velocity of the absorption component is $-4420 \mathrm{~km} \mathrm{~s}^{-1}$. Probably Fe II $42, \lambda 5169$ also may contribute to these emission and absorption features.

There are two deep absorption lines at $\lambda \lambda 4941$ and $4972 \AA$. The former could be due to N II 4, $\lambda 5010.6$ blueshifted by $-4130 \mathrm{~km} \mathrm{~s}^{-1}$, while the latter may be of N II 4 , $\lambda 5045.1$ blue-shifted by $-4310 \mathrm{~km} \mathrm{~s}^{-1}$. There also may be some contribution from He I 4, $\lambda 5015.7$ and Fe II 42, $\lambda 5018.4$ in the former and from He I 47, $\lambda 5047.7$ in the latter. In addition to these elements, He I 48, $\lambda 4921.9$ and Fe II $42, \lambda 4923.9$ may contribute to the broad emission component in the range $\lambda \lambda 4950-5080 \AA$.

The weak emission features at $\lambda \lambda 5354$ and $5280 \AA$ may be identified as the primary and secondary components of N II $69, \lambda 5321.0$ whose radial velocities are $+1830 \mathrm{~km} \mathrm{~s}^{-1}$ and $-2360 \mathrm{~km} \mathrm{~s}^{-1}$.

\subsection{From He I 4471 to $H \beta$}

Figure 5 shows the spectral region $\lambda \lambda 4350-5000 \AA$. The $F W H M$ of $\mathrm{H} \beta$ emission is $7630 \mathrm{~km} \mathrm{~s}^{-1}$. The main emission peak of $\mathrm{H} \beta$ is red-shifted by $2320 \mathrm{~km} \mathrm{~s}^{-1}$, and a weak emission peak is blue-shifted by $-3160 \mathrm{~km} \mathrm{~s}^{-1}$. A central emission peak analogous to that of $\mathrm{H} \alpha$ is not seen, whereas an absorption component blue-shifted by $-4820 \mathrm{~km} \mathrm{~s}^{-1}$ is present. A weak absorption feature at $\lambda 4850 \AA$ on the $\mathrm{H} \beta$ emission, indicated by an asterisk, could be identified as He I $48, \lambda 4921.9$ blue-shifted by about $-4310 \mathrm{~km} \mathrm{~s}^{-1}$.

It has been believed that the diffuse emission features in the range $\lambda \lambda 4600$ to $4720 \AA$ are mainly due to N III 2 , $\lambda \lambda 4634.2,4640.6$, and 4641.9 , and C III $1, \lambda \lambda 4647.4$ and 4650.2 (e.g. Barlow et al. 1981; Rosino \& Iijima 1988; Sekiguchi et al. 1988). In our spectrum the peak of the emission complex is found at $\lambda 4660 \AA$. If this emission component is red-shifted by about $2000 \mathrm{~km} \mathrm{~s}^{-1}$, as for the other emission components, its rest wavelength is about $\lambda 4630 \AA$. If the lines of N III are the main contributors to this emission complex, the rest wavelength of the peak should be found around $\lambda 4640 \AA$, because the intensity of the sum of N III $\lambda \lambda 4640.6$ and 4641.9 is higher than that of N III $\lambda 4634.2$ (Moore 1959). Sekiguchi et al. (1988) also found the rest wavelength of the peak around $\lambda 4630 \AA$, and suggested that in the ejecta of this object the emission line of N III $\lambda 4634.2$ might have been stronger than the sum of N III $\lambda \lambda 4640.6$ and 4641.9 being in conflict with the laboratory intensities. In our spectrum, however, many unusually strong emission lines of N II are detected. Probably the emission lines of N II 5, $\lambda \lambda 4613.9,4621.4$, 4630.5 and 4643.1 may contribute much to this emission complex. If the contribution from N II is larger than that from N III, the higher intensity at $\lambda 4630$ than at $\lambda 4643$ is self-evident (Moore 1959).

The red-shift of the main emission peak of He I $\lambda 4471$ is $2180 \mathrm{~km} \mathrm{~s}^{-1}$ and the blue-shift of the secondary peak is $-2340 \mathrm{~km} \mathrm{~s}^{-1}$. A weak absorption component blue-shifted by $-4320 \mathrm{~km} \mathrm{~s}^{-1}$ is seen. As seen in Table 3 , the intensity ratio of He I $\lambda 4471$ to He I $\lambda 5876$ in this spectrum is much higher than the theoretical one. There may be a contribution from $\mathrm{Mg}$ II $4, \lambda \lambda 4481.1$ and 4481.3 in this emission feature. This hypothesis is supported by the presence of an absorption feature, indicated by an asterisk, at $\lambda 4421 \AA$. If this absorption is blue-shifted by $-4300 \mathrm{~km} \mathrm{~s}^{-1}$, as of the others, its rest wavelength is about $\lambda 4485 \AA$ which roughly agrees with that of the $\mathrm{Mg}$ II lines. A strong emission line of $\mathrm{Mg}$ II at $\lambda 2800$ was detected in the IUE observations on the outburst in 1979 (Williams et al. 1981), which may also support this hypothesis.

\subsection{Different profiles of emission components of same elements}

As seen in the figures and Table 2, some emission components of the same elements have different profiles and radial velocities. For examples, the emission component of $\mathrm{H} \alpha$ has three peaks (Fig. 2), while $\mathrm{H} \beta$ shows two peaks 
(Fig. 5), and the radial velocities of the emission components of $\mathrm{N}$ II lines are different to each other. A part of the different profiles and radial velocities may be due to blendings with other emission and/or absorption lines, but some of them, e.g. $\mathrm{H} \alpha$ and $\mathrm{H} \beta$, actually seem to have different profiles.

In the earliest stage of explosion, the distributions of electron temperature and density in the ejecta may not be uniform. Under such a condition, even the emission lines of the same elements might be emitted in slightly different regions, because the transition probabilities and excitation potentials of the respective lines are different. If the shape of the ejecta is not spherically symmetric, these differences might result in the different profiles and radial velocities of the emission components.

The profile of the emission component of $\mathrm{H} \alpha$ was different from that of $\mathrm{H} \beta$ in our spectrum. However, the profile of $\mathrm{H} \alpha$ one day after (Munari et al. 1999) was interestingly rather similar to that of our $\mathrm{H} \beta$, because the central emission peak had nearly merged with the redder peak. This phenomenon might be related to the high velocity expansion of the ejecta. Detailed model calculations may be required to explain these phenomena, which is, however, beyond the scope of this article.

\section{Helium abundance}

The helium abundance is estimated from the intensity ratios He I 5876/H $\beta$ and He I 5876/ $\mathrm{H} \alpha$.

Since the emission lines of He I were prominent but no He II line was seen in our spectrum, all helium atoms may have been singly ionized. If this is the case $N(\mathrm{He}) / N(\mathrm{H})=N\left(\mathrm{He}^{+}\right) / N(\mathrm{H})$. Using the material presented by Osterbrock (1989), we have

$$
\begin{aligned}
\frac{N\left(\mathrm{He}^{+}\right)}{N(\mathrm{H})} & =0.75 \frac{I(\mathrm{HeI} 5876)}{I(\mathrm{H} \beta)} \\
& =2.1 \frac{I(\mathrm{He} 5876)}{I(\mathrm{H} \alpha)},
\end{aligned}
$$

where the electron temperature is assumed to be $10000 \mathrm{~K}$.

The ratio of the collisional excitation rate to the recombination rate of He I 5876 emission is

$$
\frac{I(\mathrm{HeI} 5876)_{\mathrm{c}}}{I(\mathrm{HeI} 5876)_{\mathrm{r}}}=0.20
$$

where Eq. (13) of Peimbert and Torres-Peimbert (1987) at the high density limit was used with $T_{\mathrm{e}}=10000 \mathrm{~K}$. We have a helium abundance by the number of atom $N(\mathrm{He}) / N(\mathrm{H})=0.15$ from the ratio of $\mathrm{He} \mathrm{I} 5876 / \mathrm{H} \beta$ and 0.16 from He I $5876 / \mathrm{H} \alpha$. The effect of the blending of N II 8, $\lambda 6482$ in $\mathrm{H} \alpha$ may be less than the observational error.

In our spectrum the intensity ratio of He I $\lambda 4471$ to He I $\lambda 5876$ is about three times higher than the theoretical one (e.g. Osterbrock 1989). If we use the observed intensity ratio He I $4471 / \mathrm{H} \beta$, we have a helium abundance of about 0.5 from the following formulae.

$\frac{N\left(\mathrm{He}^{+}\right)}{N(\mathrm{H})}=2.05 \frac{I(\mathrm{He} \mathrm{I} 4471)}{I(\mathrm{H} \beta)}$

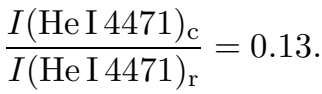

Another less accurate estimate of helium abundance was made using the emission line of He I $\lambda 6678$ whose intensity is rather uncertain because of the blending with $\mathrm{H} \alpha$. Using the intensity of He I $\lambda 6678$ in Table 3 and the following formulae, we have $N(\mathrm{He}) / N(\mathrm{H}) \simeq 0.2$.

$\frac{N\left(\mathrm{He}^{+}\right)}{N(\mathrm{H})}=4.23 \frac{I(\mathrm{He} \mathrm{I} 6678)}{I(\mathrm{H} \beta)}$,

$\frac{I(\operatorname{HeI} 6678)_{\mathrm{c}}}{I(\operatorname{HeI} 6678)_{\mathrm{r}}}=0.085$

This result supports the helium abundance derived from the He I $\lambda 5867$ line. The high intensity of the He I $\lambda 4471$ line may have been caused by the blending with the emission lines of Mg II 4, $\lambda 4481$. Here, we adopt the helium abundance $N(\mathrm{He}) / N(\mathrm{H})=0.16 \pm 0.02$ averaging the values of He I $5876 / \mathrm{H} \beta$ and $\mathrm{He} \mathrm{I} 5876 / \mathrm{H} \alpha$. This abundance is frequently found among classical novae (e.g. Truran \& Livio 1986; Snijders 1990).

Barlow et al. (1981) derived an unusually high helium abundance, $N(\mathrm{He}) / N(\mathrm{H})=2$, in the analysis of the spectra on the outburst in 1979. Their first spectra were taken eight days after maximum when the emission line of He II $\lambda 4686$ was much stronger than $\mathrm{H} \beta$, and no prominent emission lines of He I were seen. They derived the helium abundance using the intensity ratios of He II lines to H I lines. To do this, however, we have to consider the difference of the absorption coefficients of the ionizing photons for the $\mathrm{He}^{+}$ion, $\lambda \leq 228 \AA$, and for the hydrogen atom, $\lambda \leq 912 \AA$, in the nebula. Since the mass of the ejecta was only $\sim 10^{-7} M_{\odot}$ (Williams et al. 1981), the emission nebulosity probably was not ionization limited, but was matter limited when their observations were made. In such a condition the ionizing photons for hydrogen atoms more easily escape from the nebulosity than those for $\mathrm{He}^{+}$ions (see, e.g. Osterbrock 1989). Due to this effect, the emission lines of H I become relatively weak, and as a result we have apparent high helium abundances. It may be possible to explain the high helium abundance of Barlow et al. (1981) with this effect.

Recently, Anupama \& Dewangan (2000) found $N(\mathrm{He}) / N(\mathrm{H})=0.4$ for the present outburst. Their result is lower than that of Barlow et al. (1981), but is still higher than ours. Since they used spectra taken 11 and 12 days after maximum, when the emission lines of He II were very strong, their high helium abundance may be also due to the same effect.

In our analysis the emission lines of He I are used, and the emission region was surely ionization limited, because 
P Cyg type absorption features of H I and He I were seen. For these reasons our result may represent a more realistic helium abundance.

\section{Mass of ejecta}

Following Osterbrock (1989), we have a relation between the intensity of the $\mathrm{H} \beta$ emission line and the mass of hydrogen in the emitting nebula as:

$m / M_{\odot}=\frac{D^{2}}{N_{\mathrm{e}}} 7.1 \times 10^{11} I(\mathrm{H} \beta)$,

where $m$ is the mass of the hydrogen in the nebula, $D$ is distance to the object in kpc, $N_{\mathrm{e}}$ is electron density in number $\mathrm{cm}^{-3}$, and $I(\mathrm{H} \beta)$ is the intensity of $\mathrm{H} \beta$ in $\operatorname{erg} \mathrm{cm}^{-2} \mathrm{~s}^{-1}$. The effective recombination coefficient of $\mathrm{H} \beta$ at $T_{\mathrm{e}}=10000 \mathrm{~K}$ and $N_{\mathrm{e}}=10^{9} \mathrm{~cm}^{-3}$ (Hummer \& Storey 1987) is used.

Various distances have been proposed for this object, e.g. $3.5 \pm 1.5 \mathrm{kpc}$ (Hanes 1985), 3.3-8.6 kpc (Kato 1990), $14.89 \mathrm{kpc}$ (Webbink et al. 1987). Recently, Hachisu et al. (2000a) compared the light curve of the present outburst with their model of thermonuclear burning on a massive white dwarf and proposed a distance of $6 \mathrm{kpc}$. Hachisu et al. (2000b) obtained nearly the same distance also in the study of the luminosity of this object in the quiescent stage. Using the distance of $6 \mathrm{kpc}$ and the intensity of $\mathrm{H} \beta$ in Table 3, we have

$m / M_{\odot}=4.3 \times 10^{3} / N_{\mathrm{e}}$.

Another simple estimate of the mass of the ejecta was made assuming that the ejecta have a uniform density. The mass of hydrogen in the ejecta will be given as:

$m=\frac{4}{3} \pi R^{3} m_{\mathrm{H}} N_{\mathrm{e}} \epsilon$,

where $R$ is radius of the ejecta, $m_{H}$ is the mass of a hydrogen atom and $\epsilon$ is a factor that represents the filling factor of the ejecta and the geometrical covering factor.

Hachisu et al. (2000a) suggest that the mass of the white dwarf in the system of U Sco is $1.37 M_{\odot}$. The luminosity at light maximum may be close to the Eddington limit of the white dwarf. If we assume the effective temperature of the photosphere as $8000 \mathrm{~K}$, the apparent magnitude is $m_{V} \sim 8$, where we adopted $D=6 \mathrm{kpc}$ and $A_{V}=0.6$. This result is consistent with the observed ones. At the same time the radius of the photosphere is about $7.6 \times 10^{7} \mathrm{~km}$.

We assume that the radius of the ejecta at light maximum was equal to that of the photosphere. Since our first spectrum was taken about 16 hours after maximum, the outer radius of the ejecta may have been about $3.1 \times 10^{8} \mathrm{~km}$, where the expanding velocity was estimated as $4000 \mathrm{~km} \mathrm{~s}^{-1}$ from the width of $\mathrm{H} \alpha$ emission line. The variation of the inner radius of the ejecta being due to the shrinking of the photosphere may not be necessary to take into account in our rough estimate. The volume of the ejecta was about $1.2 \times 10^{26} \mathrm{~km}^{3}$ if it was spherical, and we have,

$$
m / M_{\odot}=1.0 \times 10^{-16} N_{\mathrm{e}} \epsilon .
$$

The profile of the emission component of $\mathrm{H} \alpha$ of our spectrum (Fig. 2) is more similar to that of a ring shaped emission nebula than that of a spherically symmetric nebula (see, e.g. Shore et al. 1993). It is, however, difficult to know the real shape of the ejecta soon after the outburst. So, we made two estimates, namely a spherically symmetric case $(\epsilon=1)$ and a slim ring case $(\epsilon=0.1)$. Using Eqs. (9) and (11) we have:

$N_{\mathrm{e}}=6.6 \cdot \epsilon^{-1 / 2} \times 10^{9} \mathrm{~cm}^{-3}$,

$m=6.6 \cdot \epsilon^{1 / 2} \times 10^{-7} \quad \mathrm{M}_{\odot}$.

If the ejecta was spherically symmetric $(\epsilon=1)$, we would have $N_{\mathrm{e}}=6.6 \times 10^{9} \mathrm{~cm}^{-3}$ and $m=6.6 \times 10^{-7} M_{\odot}$, while if the ejecta was a slim ring $(\epsilon=0.1)$, we would have $N_{\mathrm{e}}=2.1 \times 10^{10} \mathrm{~cm}^{-3}$ and $m=2.1 \times 10^{-7} M_{\odot}$. These high electron densities are consistent with the lack of nebular emission lines in our spectra.

As mentioned before, the helium atoms in the ejecta may have been singly ionized at that time. According to the helium abundance of 0.16 obtained in the previous section, the total mass of the ejecta should be 1.4 times higher than the mass of the hydrogen. Therefore, the total mass of the ejecta is $m_{\text {total }}=3 \times 10^{-7} M_{\odot}$ for $\epsilon=0.1$ or $9 \times 10^{-7} M_{\odot}$ for $\epsilon=1$. These results are higher than that of Williams et al. (1981), $\sim 10^{-7} M_{\odot}$, obtained on the 1979 outburst.

In the model of Hachisu et al. (2000a) the mass loss in one outburst is expected to be $1.8 \times 10^{-6} M_{\odot}$. Dr. I. Hachisu kindly informed me that the mass loss rate in their model at light maximum is $3 \times 10^{-4} M_{\odot} \mathrm{yr}^{-1}$, namely the mass lost in the first 16 hours is $6 \times 10^{-7} M_{\odot}$. Our result agrees with their model if the factor is $\epsilon=0.4$, namely if the ejecta was a fat ring.

\section{Spectra after maximum}

A medium dispersion spectrum was taken on March 8 (JD: 2451245.7), 10.6 days after maximum when the $V$ magnitude was about 13 . The spectral range was from $\lambda \lambda 4000$ to $5100 \AA$, and the resolution was $\lambda / \Delta \lambda \sim 800$. A trace of the spectrum is shown in Fig. 6, where the unit of the ordinate is $10^{-14} \mathrm{erg} \mathrm{cm}^{-2} \mathrm{~s}^{-1} \AA^{-1}$. The emission line of He II $\lambda 4686$ is stronger than $\mathrm{H} \beta$, and the emission complex around $4640 \AA$, which may be due mainly to N III 2, $\lambda \lambda 4634.2,4640.6$, and 4641.9 , is prominent. The emission feature at $\lambda 4100 \AA$ could be identified as N III $1, \lambda \lambda 4097.3$ and 4103.4. These spectral features are similar to those of the spectra taken on July 2 and 3, 1979 (Barlow et al. 1981), on May 21.87, 1987 (Sekiguchi et al. 1988) and on May 22, 1987 (Rosino \& Iijima 1988). All these observations were made when $V$ mag was about 13 . 


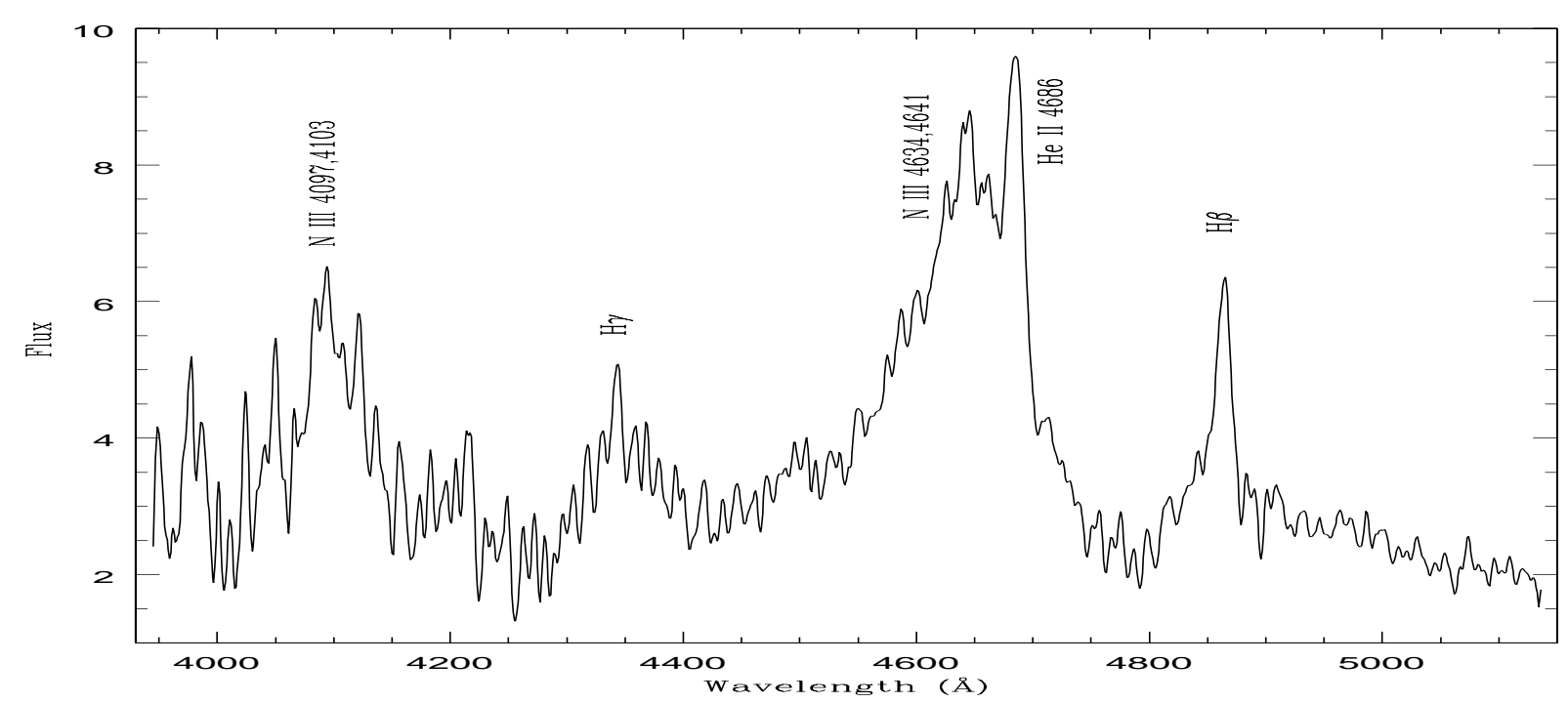

Fig. 6. The medium dispersion spectrum taken on March 8.2 UT, 11 days after maximum. The unit of the ordinate is $10^{-14} \mathrm{erg} \mathrm{cm}^{-2} \mathrm{~s}^{-1} \AA^{-1}$.

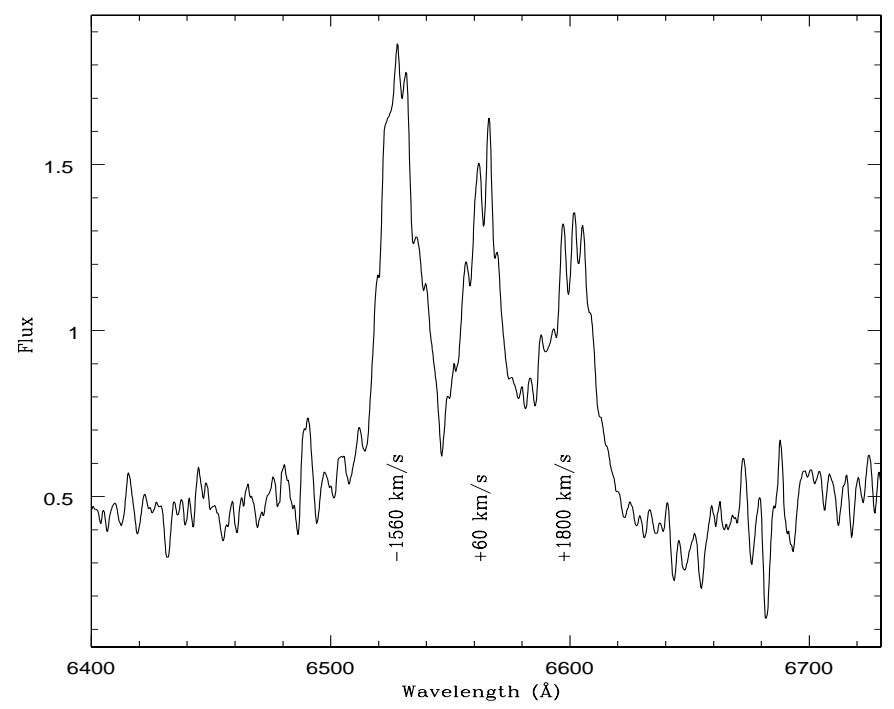

Fig. 7. $\mathrm{H} \alpha$ region of the high dispersion spectrum taken on March 14.1 UT, 17 days after maximum. The unit of the ordinate is $10^{-14} \mathrm{erg} \mathrm{cm}^{-2} \mathrm{~s}^{-1} \AA^{-1}$.

Another high dispersion spectrum was taken on March 14.17 UT (JD: 2451251.67), when the $V$ magnitude was about 14. Figure 7 shows the emission feature of $\mathrm{H} \alpha$, where the unit of the ordinate is $10^{-14} \mathrm{erg} \mathrm{cm}^{-2} \mathrm{~s}^{-1} \AA^{-1}$. H $\alpha$ again has three emission components in this spectrum, but the full width and the widths of the individual components have significantly decreased with respect to those of the first spectrum. The radial velocities of these peaks are $-1560 \mathrm{~km} \mathrm{~s}^{-1},+60 \mathrm{~km} \mathrm{~s}^{-1}$ and $+1800 \mathrm{~km} \mathrm{~s}^{-1}$, respectively and the $F W H M$ is about $4060 \mathrm{~km} \mathrm{~s}^{-1}$. In contrast to the first spectrum, the blue peak is more intense than the others. The narrowing of the prominent emission lines with time is reported also in other works (Munari et al. 1999; Anupama \& Dewangan 2000).

Figure 8 shows the spectral range $\lambda \lambda 4600-5000 \AA$. $\mathrm{H} \beta$ also has three peaks of which radial velocities are

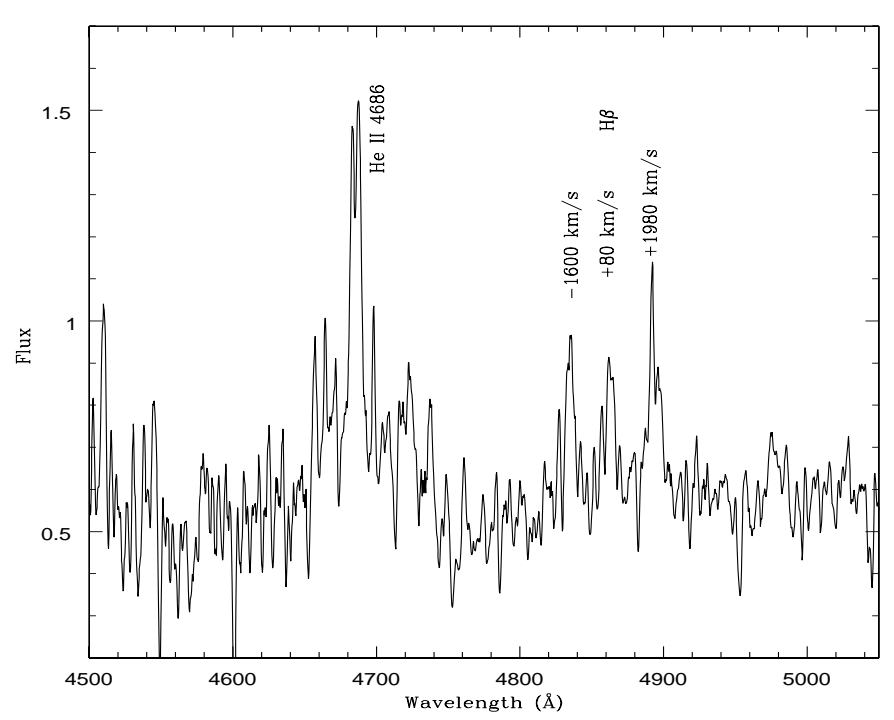

Fig. 8. The spectral region $4500-5100 \AA$ of the spectrum of 17 days after maximum.

$-1600 \mathrm{~km} \mathrm{~s}^{-1},+80 \mathrm{~km} \mathrm{~s}^{-1}$ and $+1980 \mathrm{~km} \mathrm{~s}^{-1}$, respectively. The emission line of He II $\lambda 4686$, which is stronger than $\mathrm{H} \beta$, seems to have no satellite emission component, or at least the satellite components are very weak. The central emission component of He II 4686 splits into two peaks the radial velocities of which are $160 \mathrm{~km} \mathrm{~s}^{-1}$ and $-120 \mathrm{~km} \mathrm{~s}^{-1}$.

\section{Concluding remarks}

Since the outbursts of U Sco fade very rapidly, the spectrum near maximum was not observed in the previous outbursts. Fortunately we were able to make a spectroscopic observation near maximum of the outburst in February 1999 by the help of the world wide astronomical networks. The spectrum near maximum shows significantly different features with respect to those observed in the 
previous works. For example, in addition to the known prominent emission lines of H I, He I, and N II, our spectrum shows largely blue-shifted narrow absorption components of these elements which were not noticed in the previous observations. Since the absorptions disappeared over one day, detailed observations as early as possible are very important in the study of the outbursts of this object. As seen in Sect. 3, the absorption components could be good clues for the line identifications.

The helium abundance, $N(\mathrm{He}) / N(\mathrm{H})=0.16 \pm 0.02$, is less than one tenth of that derived by Barlow et al. (1981), and is not different from those of usual classical novae. This result seems to require us to change our interpretation of the outbursts of this object.

Acknowledgements. I am grateful to Profs. I. Hachisu and M. Kato for the useful discussions and comments, and to Prof. R. Barbon for the careful reading of the manuscript and useful suggestions. Thanks are also due to Dr. T. Kato, Dr. D. Nogami, Mr. S. Kiyota, and many other professional and amateur astronomers who supplied photometric data of U Sco on the VS-Net.

\section{References}

Anupama, G. C., \& Dewangan, G. C. 2000, AJ, 119, 1359

Barlow, M. J., Brodie, J. P., Brunt, C. C., et al. 1981, MNRAS, 195, 61

Bonifacio, P., Molaro, P., Śelvelli, P. 1999, IAU Circ., 7129

Hachisu, I., Kato, M., Kato, T., \& Matsumoto, K. 2000a, ApJ, 528, L97

Hachisu, I., Kato, M., Kato, T., Matsumoto, K., \& Nomoto, K. 2000b, ApJ, 534, L189

Hanes, D. A. 1985, MNRAS, 213, 443

Hummer, D. G., \& Storey, P. J. 1987, MNRAS, 224, 801
Iijima, T. 2000, New Astron. Rev., 44, P63

Kahabka, P., Hartmann, H. W., Parmar, A. N., \& Negueruela, I. 1999, A\&A, 347, L43

Kato, M. 1990, ApJ, 355, 277

Lèpine, S., Shara, M. M., Livio, M., \& Zurek, D. 1999, ApJ, 522, L121

Mattiazzo, M. 1999, IAU Circ., 7113

Moore, C. E. 1959, A Multiplet Table of Astrophysical Interest, US Dept. of Commerce (Office of Technical Services, Washington DC)

Munari, U., Zwitter, T., Tomov, T., et al. 1999, A\&A, 347, L39

Niedzielski, A., Tomov, T., \& Munari, U. 1999, IAU Circ., 7115

Osterbrock, D. E. 1989, Astrophysics of Gaseous Nebulae and Active Galactic Nuclei (University Science Books, Mill Valley, CA)

Peimbert, M., \& Torres-Peimbert, S. 1987, Rev. Mex. Astron. Astrofís., 14, 540

Rosino, L., \& Iijima, T. 1988, A\&A, 201, 89

Schaefer, B. E., \& Ringwald, F. A. 1995, ApJ, 447, L45

Schmeer, P. 1999, IAU Circ., 7113

Sekiguchi, K., Feast, M. W., Whitelock, P. A., et al. 1988, MNRAS, 234, 281

Shaw, L. 1999, IAU Circ., 7113

Shore, S. N., Sonneborn, G., Starrfield, S., Gonzalez-Riestra, R., \& Ake, T. B. 1993, AJ, 106, 2408

Snijders, M. A. J. 1990, in Physics of Classical Novae, ed. A. Cassatella, \& R. Viotti (Springer-Verlag), 188

Starrfield, S., Aparks, W. M., \& Truran, J. W. 1985, ApJ, 291, 136

Truran, J. W., \& Livio, M. 1986, ApJ, 308, 721

Webbink, R. F., Livio, M., Truran, J. W., \& Orio, M. 1987, ApJ, 314, 653

Williams, R. E., Sparks, W. M., Gallagher, J. S., et al. 1981, ApJ, 251, 221

Zwitter, T., \& Munari, U. 1999, IAU Circ., 7118 